\title{
Dynamic Interpolation of Sea Surface Height and Potential Applications for Future High-Resolution Altimetry Mapping
}

\author{
Clement UbelmanN \\ Jet Propulsion Laboratory, California Institute of Technology, Pasadena, California \\ PATRICE KLEIN \\ Laboratoire de Physique des Océans, IFREMER, Plouzané, France \\ LEE-LUENG FU \\ Jet Propulsion Laboratory, California Institute of Technology, Pasadena, California
}

(Manuscript received 1 August 2014, in final form 15 October 2014)

\begin{abstract}
Many issues may challenge standard interpolation techniques to produce high-resolution gridded maps of sea surface height in the context of future missions like Surface Water and Ocean Topography (SWOT). The present study proposes a new method to address these challenges. Based on the conservation of potential vorticity, the method provides a simple dynamic approach to interpolation through temporal gaps between high spatial resolution observations. For gaps shorter than 20 days, the dynamic interpolation is extremely efficient and allows for the reconstruction of the time evolution of small mesoscale eddies (below $100 \mathrm{~km}$ ) that would be smoothed out by conventional methods based on optimal mapping. Such a simple approach offers some perspectives for developing high-level products from high-resolution altimetry data in the future.
\end{abstract}

\section{Introduction}

The measurement of wide-swath sea surface height (SSH) planned for future space missions [e.g., the Surface Ocean and Water Topography (SWOT) Mission; see Fu and Ferrari 2008; Durand et al. 2010) promises to observe the ocean mesoscale eddies at unprecedented spatial resolutions, reaching $15-\mathrm{km}$ wavelength in most parts of the global oceans owing to the very low noise floor of the two-dimensional measurements. However, the temporal resolution (i.e., the time between satellite revisits of the order of 10 days for a complete repeat cycle of 21 days) may not be sufficient to continuously capture the evolution of small eddies. The mismatch between the high spatial resolution and the moderate temporal resolution of the satellite presents a challenge for constructing time-continuous maps of SSH.

Corresponding author address: Clement Ubelmann, Jet Propulsion Laboratory, California Institute of Technology, 4800 Oak Grove Dr., Pasadena, CA 91109.

E-mail: clement.ubelmann@jpl.nasa.gov
Presently, the most commonly used maps of SSH, produced by the AVISO data center are based on optimal mapping in time and space from multiple satellite nadir observations (e.g., Le Traon and Dibarboure 1999; Ducet and Le Traon 2000; Morrow and Le Traon 2012). The reconstruction of SSH maps from future SWOT data with such methods has been evaluated in Pujol et al. (2012). Within the swath of SWOT, its two-dimensional mapping capability significantly outperforms that of the combination of four conventional altimeters. However, owing to the limited temporal coverage of SWOT, its overall mapping capability is equivalent to only two to four conventional altimeters depending on the geographic locations of varying sampling characteristics. This apparent poor performance is probably caused by the inability of the mapping technique to utilize some dynamic properties of the ocean to mitigate the effects of poor temporal sampling. The objectives of the present study are first, to highlight the deficiency of the standard optimal interpolation methods in the context of SWOT; and second, to explore the concept of dynamic interpolation (DI) to mitigate the shortcomings of large time gaps. 

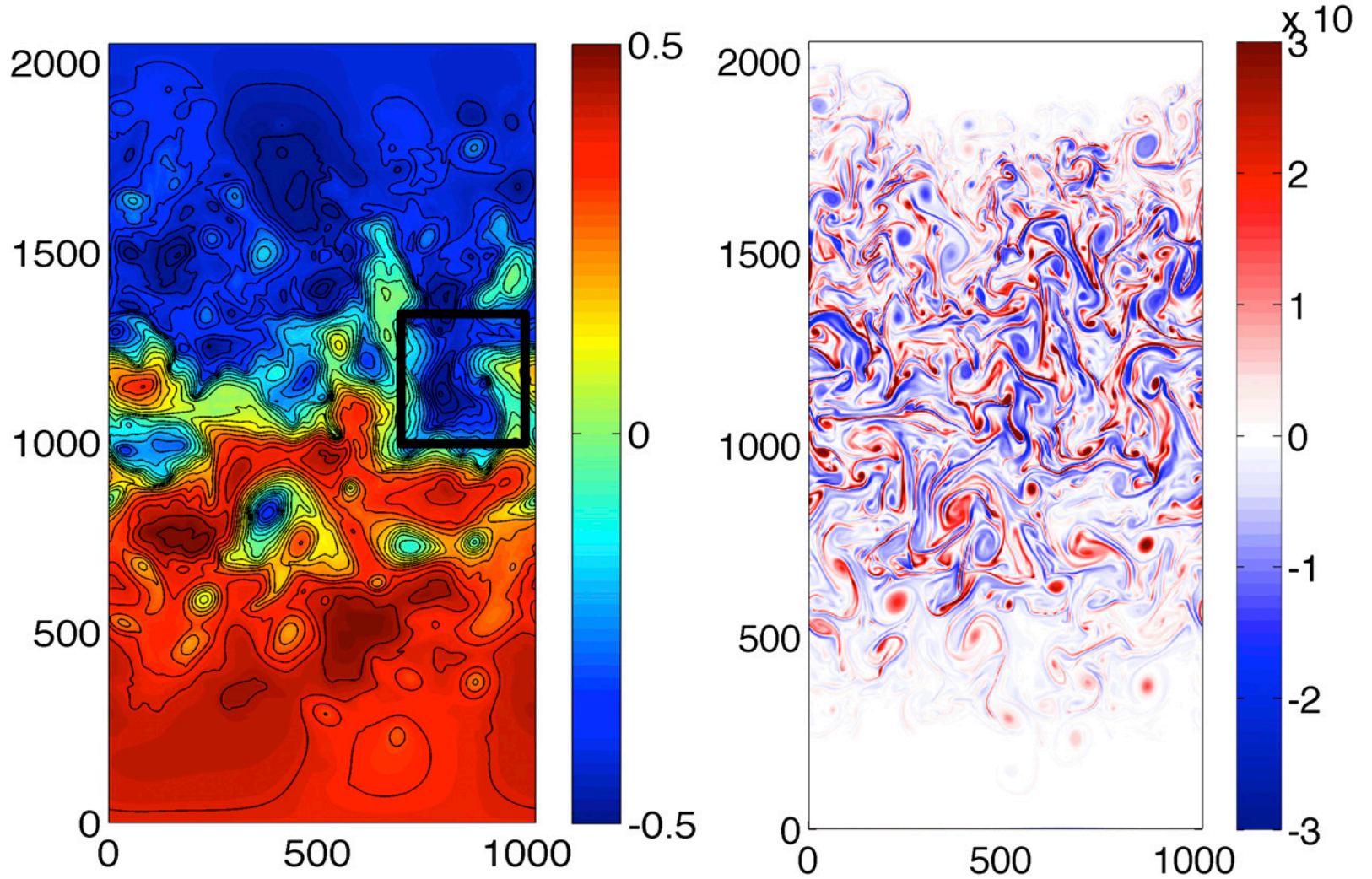

FIG. 1. (left) SSH (m) and (right) relative vorticity $\left(\mathrm{s}^{-2}\right)$ over the $1000 \mathrm{~km} \times 2000 \mathrm{~km}$ domain of the reference model simulation. The domain is periodic along the $x$ axis.

Without dealing with the actual sampling characteristics and measurement errors of SWOT, which would require further development of an inverse problem, this study illustrates the potential to increase mapping capabilities from simple dynamic considerations of SSH images separated in time.

In this study, the outputs from a high-resolution numerical model described in section 2 are taken as the true state of the ocean. Section 3 illustrates the difficulties in applying standard interpolation methods to highresolution SSH fields separated by large time gaps. Then, the new DI method is presented (section 4) with results discussed in section 5. Finally, the potential applications for future high-resolution altimetry missions and conclusions are presented in section 6.

\section{Experimental setup}

The synthetic ocean state used in the study comes from a high-resolution numerical simulation of the mesoscale and submesoscale ocean circulation in a betaplane channel model formulated with the primitive equations. The horizontal resolution is $2 \mathrm{~km}$ with 100 vertical levels involving depth intervals of about $3 \mathrm{~m}$ near the surface. The simulation, described in Klein et al. (2008), consists of an unstable westerly zonal flow in a domain size of $1000 \mathrm{~km} \times 2000 \mathrm{~km}$ with a depth of $4000 \mathrm{~m}$. The resulting turbulence is intensified at the surface, where the submesoscale dynamics is active and the wavenumber spectrum of horizontal kinetic energy is shallow ( $k^{-2}$, where $k$ is the wavenumber). The Rossby number (defined as the ratio of the RMS value of the relative vorticity to the planetary vorticity) is close to 0.6. The first Rossby radius of deformation is of the order of $25 \mathrm{~km}$ (or $\sim 160 \mathrm{~km}$ in terms of wavelength). More details can be found in Klein et al. (2008) and Pujol et al. (2012).

Figure 1 shows a snapshot of the SSH (right) and relative vorticity (left) in the channel domain. The SSH drop between the southern and northern parts of the turbulent eddy field is about $0.8 \mathrm{~m}$. Maximal surface velocities attain $1.5 \mathrm{~m} \mathrm{~s}^{-1}$ (not shown), which are typical values for the western boundary currents. The $2-\mathrm{km}$ resolution allows the representation of the submesoscale structures $O(10 \mathrm{~km})$ associated with the intense fronts and filaments, particularly in the vorticity field. Two months' worth of integration constitutes the simulated database for the present study. Snapshots of SSH have been extracted at a 12 -h frequency. 

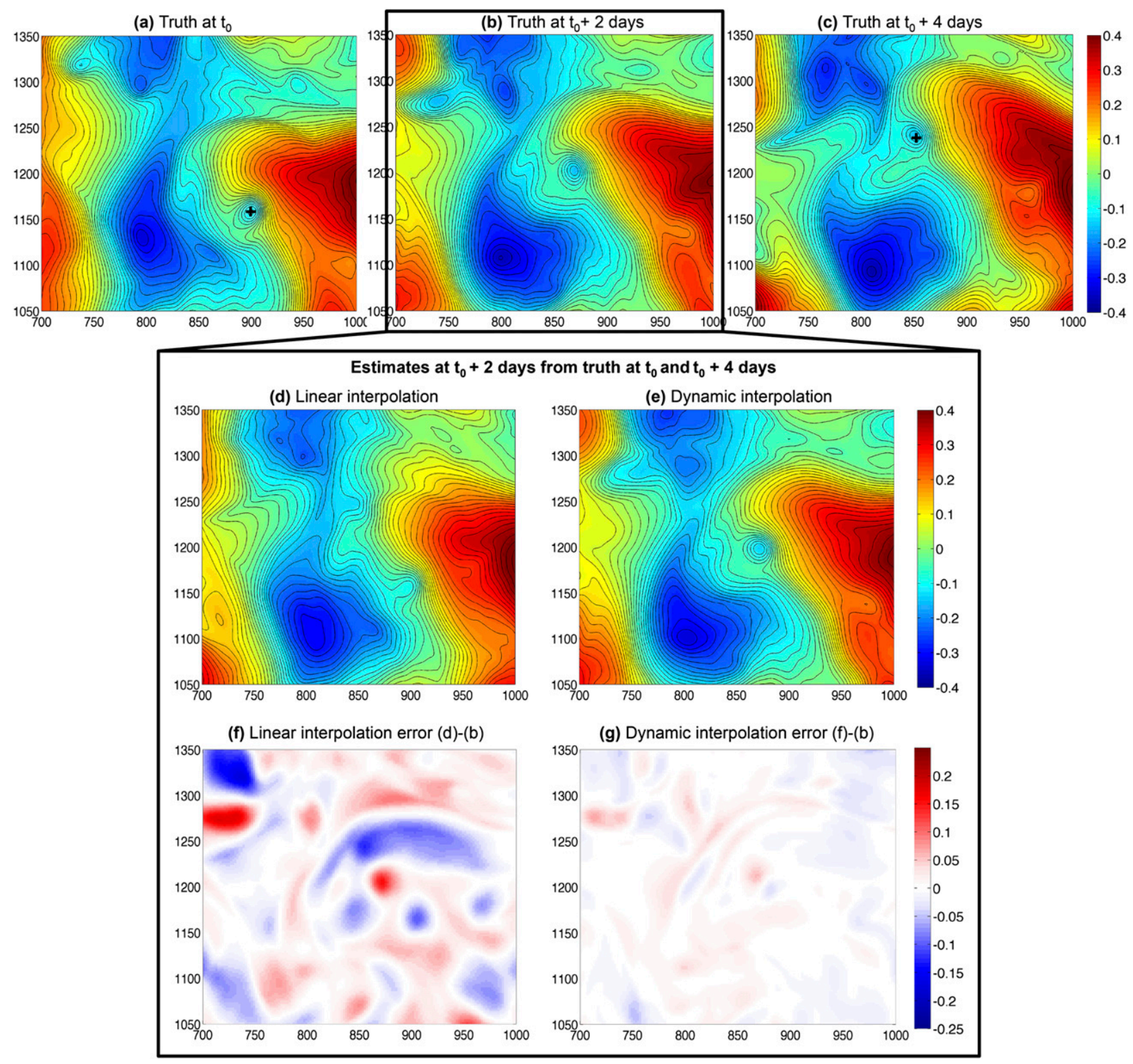

FIG. 2. (a)-(c) SSH in the rectangle shown in Fig. 1 at 2-day intervals. (d) Linear estimation of (b) from (a) and (c). (e) Dynamic estimation of (b) from (a) and (c). (f) Difference between (d) and (b). (g) Difference between (e) and (b). Units are in meters.

\section{Future challenges for high-resolution altimetry mapping}

The large eddies resolved by conventional altimetry mapping ( $>150-\mathrm{km}$ wavelength) involve relatively slow motions compared to the repeat periods of the satellites. Large mesoscale eddies evolve at time scales on the order of a month, while satellites have generally shorter repeat periods (e.g., Jason class has a 10-day repeat). These eddies are therefore almost continuously sampled both in space and time. In this context, the constellation of altimeters has allowed the generation of accurate maps of the SSH evolution for large mesoscale eddies, such as the one produced by AVISO (e.g., Le Traon et al. 1998; Morrow and Le Traon 2012). However, smaller mesoscale eddies expected to be seen by future altimetry missions behave differently with fairly shorter time scales. This is illustrated in Figs. 2a-c: in this example, during 4 days, the eddy marked by the black cross in Figs. 2a,c travels about $70 \mathrm{~km}$, which is about twice its diameter. The turbulent dynamics involving eddies of different scales is well documented in the literature (Rhines 1975; McWilliams 1984, 1985; Hua and Haidvogel 1986): eddy structures embedded in a turbulent flow are known to be advected, stirred, and deformed by the velocity field of larger scales, eventually leading to 
smaller scales. They can also merge with other eddies, leading to larger eddies. These features are known as the direct cascade of potential enstrophy and inverse cascade of kinetic energy (Hua and Haidvogel 1986). The time scale of these cascade dynamics is usually of the order of one day, much smaller than the time scale of large eddies (McWilliams 1985; Hua 1994; Klein and Hua 1990). This short time scale is the one associated with the small eddies with a size of $10-50 \mathrm{~km}$ (Roullet and Klein 2010). The existence of these small scales usually leads to an SSH spectrum with a slope of $k^{-4}$ instead of $k^{-5}$ (Capet et al. 2008; Klein et al. 2008). Recent observations (Le Traon et al. 2008; Lumpkin and Elipot 2010; Xu and Fu 2012; Poje et al. 2014) demonstrate the presence and dynamical impact of these small features that are not correctly sampled and detected by conventional altimeters.

In mapping the SSH continuously in time, the standard interpolation methods would filter out most of the small-scale eddies. To illustrate this point, we display in Fig. $2 \mathrm{~d}$ a simple linear interpolation (LI) between the two fields at $t_{0}$ and $t_{0}+4$ days, respectively. The linear interpolation obviously misrepresents the small eddy that is decomposed into two attenuated eddies at the positions where the actual eddy was present at $t_{0}$ and at $t_{0}+4$ days, respectively. We also applied the optimal interpolation method (as defined in Bretherton et al. 1976) between the two SSH fields. With the time and space decorrelation coefficients commonly used for mesoscale mapping $(100 \mathrm{~km}$ in space and 10 days in time), the solution is very close to the linear estimate (not shown). By reducing the time decorrelation, we have been able to slightly smooth the small eddy misrepresented in the linear estimate, but the residual error variance is almost unchanged. For these reasons, we consider that the linear estimate gives a fair representation of the performance of the standard optimal interpolation method. This assumption is reasonable only in this specific case of interpolation between two images free of observation errors.

From this illustration, it is clear that standard optimal interpolation methods would fail to reconstruct small mesoscale eddies if they are not continuously sampled in time. Even if the eddies experience very basic transformationlike advections (which is the case for the small eddy in Fig. 2), their displacement would be seen as decorrelation. Though some recent studies have been aimed at improving the mapping of finescale features (e.g., Dussurget et al. 2011; Escudier et al. 2013), such a method still cannot take into account flow-induced structure displacement. In the context of the next generation of high-resolution altimeters, standard optimal interpolation methods would therefore not be able to take full advantage of the high spatial resolution of observations to produce accurate maps of the ocean eddy field.

\section{The dynamic interpolation approach}

A large part of the mesoscale turbulent dynamics and associated energy cascades mentioned before can be captured by the potential vorticity (PV) equation within the quasigeostrophic (QG) dynamical framework as long as the Rossby number is smaller than 1 .

In this study, we use the simplest PV equation: because of the short time scales considered (fewer than 20 days), the mixing and forcing terms are omitted as a first approximation. We consider only scales up to those corresponding to the velocity spectral peak $(\sim 300 \mathrm{~km})$. These scales are usually smaller than the Rhines scale, for which the beta effect becomes nonnegligible (see Vallis 2006, chapters 11 and 12). Consequently, this effect is not taken into account and therefore the Coriolis parameter is assumed to be constant within the turbulent field considered. We further assume that the QG dynamics-and therefore $\mathrm{SSH}$ - is principally captured by the first baroclinic mode (Fu and Flierl 1980; Shafer Smith and Vallis 2001). The resulting simplified equation is

$$
\frac{\partial q}{\partial t}+J(\psi, q)=0
$$

where $J$ is the Jacobian operator and $\psi$ is the geostrophic streamfunction. Term $q$ is the potential vorticity proportional to the first eigenmode of the Sturm-Liouville problem (e.g., Hua and Haidvogel 1986): its resulting reduced expression is

$$
q=\nabla^{2} \psi-\frac{1}{L_{R}^{2}} \psi
$$

where $\nabla^{2}$ is the horizontal 2D Laplacian operator and $L_{R}$ is the first Rossby radius of deformation. The streamfunction is related to SSH (Shafer Smith and Vallis 2001) by

$$
\psi=\frac{g}{f} \mathrm{SSH},
$$

where $g$ is the gravity constant and $f$ is the Coriolis parameter. The first Rossby radius of deformation corresponds to the one of the simulations: $L_{R}=25 \mathrm{~km}$. This value is typical of oceanic values at midlatitudes (Chelton et al. 1998).

Since mixing is neglected, Eq. (1) is reversible and therefore can be integrated, both forward and backward in time. This property is most convenient for the interpolation problem that we try to solve.

The numerical 2D setting (involving a $\mathrm{C}$ grid) is the one used in the Regional Oceanic Modeling System (ROMS; Shchepetkin and McWilliams 2005). The 

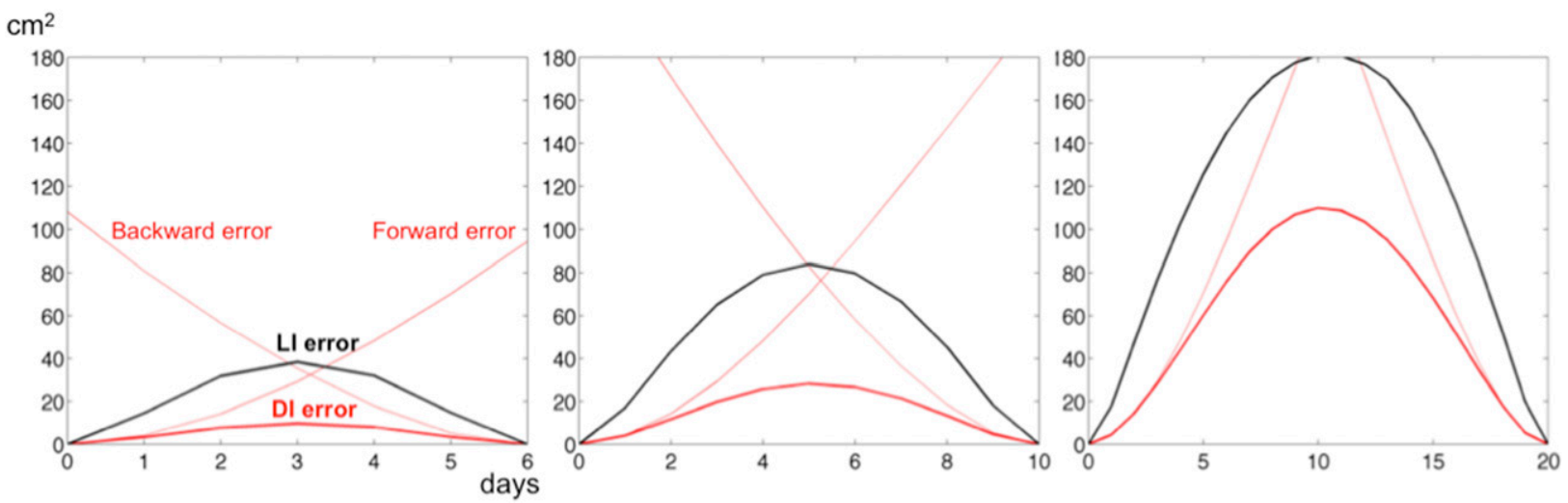

FIG. 3. Mean variance of the residual error ( $y$ axis) of the LI estimate (thick black) and the DI estimate (thick red) over three different time windows considered $(6,10$, and 20 days on the $x$ axis). The red thin curves are the residual errors of the forward and backward estimates of DI.

integration procedure is as follows: The potential vorticity $q$ is initialized with Eqs. (2) and (3) using SSH data at a given time. Then, Eq. (1) is integrated in time. At each time step, the streamfunction $\psi$ is recovered from $q$ through the inversion of Eq. (2). Inversion is performed with a conjugate gradient method with specified boundary conditions, converging after a few iterations. At any time, $\mathrm{SSH}$ is given by Eq. (3). This model basically performs an active advection of PV by geostrophic velocity.

The DI method for SSH is based on Eqs. (1)-(3) using the reversible property of Eq. (1). Let us consider two SSH fields observed at time $t_{0}$ and $t_{1}, \mathrm{SSH}_{0}$ and $\mathrm{SSH}_{1}$, separated by a time interval $T=t_{1}-t_{0}$. Equation (1) [with Eqs. (2) and (3)] is first integrated forward in time using $\mathrm{SSH}_{0}$ as the initial condition. This allows to get $\mathrm{SSH}_{f}(t)$ at different times $t$ between $t_{0}$ and $t_{1}$. Then Eq. (1) is integrated backward in time using $\mathrm{SSH}_{1}$ as the initial condition, which allows to get $\mathrm{SSH}_{b}(t)$ at different times $t$ between $t_{1}$ and $t_{0}$. The dynamic interpolated field is simply $\mathrm{SSH}_{i}(t)=\left[\mathrm{SSH}_{f}(t)+\mathrm{SSH}_{b}(t)\right] / 2$.

\section{Results}

\section{a. Residual error reduction}

Figure 2e shows the DI estimate of the $\mathrm{SSH}$ at $t_{0}+2$ days, to be compared with the estimates from a standard interpolation technique in Fig. 2d. The small eddy is now represented with a very similar amplitude, size, and position as in the true field in Fig. 2b. Figures 2f,g show the residual error with LI and DI, respectively. The improvements of DI upon LI are very clear.

The DI has also been tested over different time windows. The diagnostics have been performed over the entire domain $(1000 \mathrm{~km} \times 2000 \mathrm{~km})$ to ensure a reasonable significance. Results are shown in the three panels of Fig. 3. In this figure, the variance of the error is represented as a function of time during time windows of 6 , 10 , and 20 days, which are among typical time revisits for SWOT. The black thick lines in Fig. 3 are the variance for LI, and the red thick curves are for DI. The red thin lines present the variance of the forward and backward estimates separately. In these figures, the relative error reduction from LI to DI is demonstrated. Also, the error reduction from the single forward or backward estimate to the DI (combined) estimate is very important, as shown by the comparison between the red thin and red thick curves in Fig. 3. As the time window increases, we note that the relative gain from LI to DI becomes less important. For a 20-day time window, DI still performs better than LI by $40 \%$, while it was $\sim 80 \%$ for a 6 -day time window.

\section{b. Performances in the spectral domain}

The diagnostics presented above do not distinguish between the different spatial scales over which the performances may vary. Indeed, the variance of the error reflects the integration of the error over all scales, giving more weight to large scales, since the SSH spectrum is steep (with a slope close to $k^{-11 / 3}$ ). To examine the performance as a function of scale, we have computed the spectral coherence between the true state and the estimates (LI and DI). The spectral coherence, denoted by $C$, represents the correlation between two signals as a function of wavelength (see Klein et al. 2004; Ponte et al. 2013). It is defined as follows (for LI):

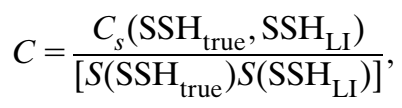

where $C_{s}$ is the cross-spectral density and $S$ is the spectral density. Results are shown in Fig. 4 for the different 


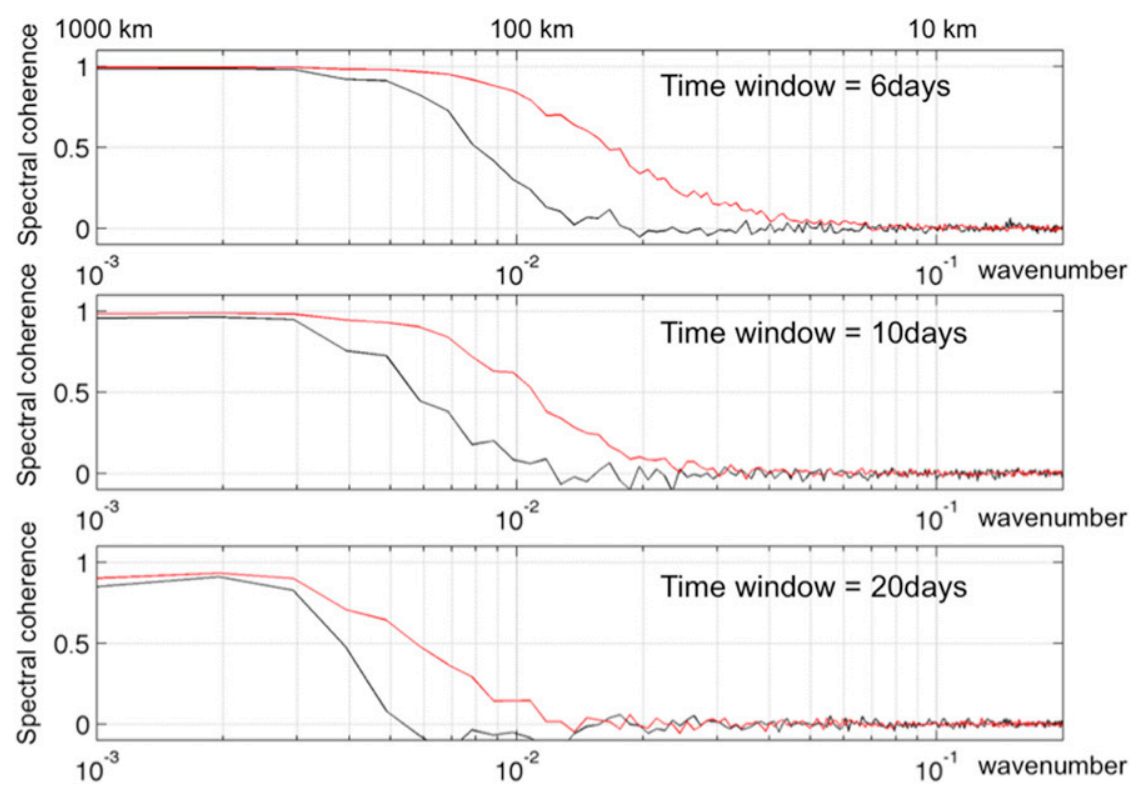

FIG. 4. Spectral coherence of the estimates (LI in black and DI in red) with the reference state at intermediate time for a 10-day time window. The $x$ axis is wavenumber and the $y$ axis is coherence (no units).

time windows considered at intermediate time when the error is the largest. The black line is the spectral coherence between the true state and LI, and the red line is the spectral coherence between the true state and DI. If we consider 0.5 as a threshold for reasonable performance, we observe that for a 10-day window, LI performs well for a wavelength down to $\sim 180 \mathrm{~km}$, while DI performs well down to $\sim 80 \mathrm{~km}$. This indicates the strong efficiency of this very simple DI method, where the potential can certainly be much improved.

\section{Discussion and conclusions}

This study has demonstrated the promise of a new interpolation scheme based on the framework of PV conservation. Assumptions leading to the PV equation have allowed to fully exploit the reversibility property for both forward and backward integration. The resulting dynamic interpolation method leads to the estimation of SSH during the time gaps of observations. This method is self-consistent, since the surface streamfunction (and therefore horizontal motions), as well as $\mathrm{SSH}$, is retrieved at any time by integrating the PV.

We could improve the physics of the dynamic interpolation. So far, only the first vertical baroclinic mode with QG approximation has been investigated for its simplicity. A straightforward improvement would involve a larger number of vertical modes, as proposed in Ponte and Klein (2013). Such development might use $\mathrm{SSH}$ and some proxy for the surface density (such as sea surface temperature observations) for the initialization of the PV (see also Lapeyre and Klein 2006).

In the context of the future wide-swath altimetry, the results presented above may hold some prospects for mitigating the impact of the long time revisits of the satellite. This study represents an idealized case, as the complex geometry of the satellite swaths and orbit sampling are not taken into account (see Pujol et al. 2012). The study specifically focuses on the temporal scheme of the interpolation using idealistic fields of SSH free of instrumental noise. Also, the reference model providing the "true ocean" did not represent unbalanced physics such as internal tides that could have a nonnegligible effect on the SSH at small scales (Chavanne and Klein 2010), particularly in the eastern parts of oceanic basins (Richman et al. 2012). For applications to the SWOT data, more sophisticated methods have to be developed. One may implement a "dynamic optimal interpolation" (DOI) scheme. Similar to the optimal interpolation (OI), the DOI would minimize the misfits with observations, but the misfits would be defined by the difference between the observation and the estimate involving signal propagation determined by the PV conservation. This is an inverse problem that may be solved with variational methods.

The development of DI (or DOI as described above) is principally motivated by the discrepancy between space and time resolution of the future wide-swath altimetry. However, the application of such algorithms to the present constellation of nadir satellites might be possible and worthy of investigation. 
Acknowledgments. The authors thank Aurelien Ponte of IFREMER, Julien Le Sommer of CNRS, and Jeroen Molemaker of UCLA for the constructive discussions about this study. The research presented in the paper was partially carried out at the Jet Propulsion Laboratory, California Institute of Technology, under contract with the National Aeronautics and Space Administration. Support from the SWOT project is acknowledged. P. K. further acknowledges the support of IFREMER (through the MOU IFREMER-JAMSTEC), CNRS (France), and the Agence Nationale de la Recherche [Contracts ANR-09-BLAN-0365-02 (REDHOT) and ANR-10-LABX-19-01 (LabexMER)]. Government sponsorship is acknowledged.

\section{REFERENCES}

Bretherton, F. P., R. E. Davis, and C. B. Fandry, 1976: A technique for objective analysis and design of oceanographic experiments applied to MODE-73. Deep-Sea Res. Oceanogr. Abstr., 23, 559-582, doi:10.1016/0011-7471(76)90001-2.

Capet, X., J. C. McWilliams, M. J. Molemaker, and A. F. Shchepetkin, 2008: Mesoscale to submesoscale transition in the California Current system. Part I: Flow structure, eddy flux, and observational tests. J. Phys. Oceanogr., 38, 29-43, doi:10.1175/ 2007JPO3671.1.

Chavanne, C. P., and P. Klein, 2010: Can oceanic submesoscale processes be observed with satellite altimetry? Geophys. Res. Lett., 37, L22602, doi:10.1029/2010GL045057.

Chelton, D. B., R. A. deSzoeke, M. G. Schlax, K. El Naggar, and N. Siwertz, 1998: Geographical variability of the first baroclinic Rossby radius of deformation. J. Phys. Oceanogr., 28, 433-460, doi:10.1175/1520-0485(1998)028<0433:GVOTFB > 2.0.CO;2.

Ducet, N., and P.-Y. Le Traon, 2000: Global high-resolution mapping of ocean circulation from TOPEX/Poseidon and ERS-1 and -2. J. Geophys. Res., 105, 19477-19498, doi:10.1029/ 2000JC900063.

Durand, M., L.-L. Fu, D. Lettenmaier, D. Alsdorf, E. Rodriguez, and D. Esteban-Fernandez, 2010: The Surface Water and Ocean Topography Mission: Observing terrestrial surface water and oceanic submesoscale eddies. Proc. IEEE, 98, 766779, doi:10.1109/JPROC.2010.2043031.

Dussurget, R., F. Birol, R. Morrow, and P. De Mey, 2011: Fine resolution altimetry data for a regional application in the Bay of Biscay. Mar. Geod., 34, 447-476, doi:10.1080/ 01490419.2011.584835.

Escudier, R., J. Bouffard, A. Pascual, P.-M. Poulain, and M.-I. Pujol, 2013: Improvement of coastal and mesoscale observation from space: Application to the northwestern Mediterranean Sea. Geophys. Res. Lett., 40, 2148-2153, doi:10.1002/grl.50324.

Fu, L. L., and G. R. Flierl, 1980: Nonlinear energy and enstrophy transfers in a realistically stratified ocean. Dyn. Atmos. Oceans, 4, 219-246, doi:10.1016/0377-0265(80)90029-9.

_ cesses from space. Eos, Trans. Amer. Geophys. Union, 89, 488, doi:10.1029/2008EO480003.

Hua, B. L., 1994: Skewness of the generalized centrifugal force divergence for a joint normal distribution of strain and vorticity components. Phys. Fluids, 6A, 3200-3202, doi:10.1063/1.868101.
- and D. B. Haidvogel, 1986: Numerical simulations of the vertical structure of quasi-geostrophic turbulence. J. Atmos. Sci., 43, 2923-2936, doi:10.1175/1520-0469(1986)043<2923: NSOTVS $>2.0 . \mathrm{CO} ; 2$.

Klein, P., and B. L. Hua, 1990: The mesoscale variability of the sea surface temperature: An analytical and numerical model. J. Mar. Res., 48, 729-763.

—, G. Lapeyre, and W. G. Large, 2004: Wind ringing of the ocean in presence of mesoscale eddies. Geophys. Res. Lett., 31, L15306, doi:10.1029/2004GL020274.

_ B. L. Hua, G. Lapeyre, X. Capet, S. Le Gentil, and H. Sasaki, 2008: Upper ocean turbulence from high-resolution 3D simulations. J. Phys. Oceanogr., 38, 1748-1763, doi:10.1175/ 2007JPO3773.1.

Lapeyre, G., and P. Klein, 2006: Dynamics of the upper oceanic layers in terms of surface quasigeostrophy theory. J. Phys. Oceanogr., 36, 165-176, doi:10.1175/JPO2840.1.

Le Traon, P.-Y., and G. Dibarboure, 1999: Mesoscale mapping capabilities of multiple-satellite altimeter missions. J. Atmos. Oceanic Technol., 16, 1208-1223, doi:10.1175/ 1520-0426(1999)016<1208:MMCOMS > 2.0.CO;2.

__, F. Nadal, and N. Ducet, 1998: An improved mapping method of multisatellite altimeter data. J. Atmos. Oceanic Technol., 15, 522-534, doi:10.1175/1520-0426(1998)015<0522: AIMMOM $>2.0 . \mathrm{CO} ; 2$.

— , P. Klein, B.-L. Hua, and G. Dibarboure, 2008: Do altimeter wavenumber spectra agree with the interior or surface quasigeostrophic theory? J. Phys. Oceanogr., 38, 1137-1142, doi:10.1175/2007JPO3806.1.

Lumpkin, R., and S. Elipot, 2010: Surface drifter pair spreading in the North Atlantic. J. Geophys. Res., 115, C12017, doi:10.1029/ 2010JC006338.

McWilliams, J. C., 1984: The emergence of isolated coherent vortices in turbulent flow. J. Fluid Mech., 146, 21-43, doi:10.1017/ S0022112084001750.

- 1985: Submesoscale, coherent vortices in the ocean. Rev. Geophys., 23, 165-182, doi:10.1029/RG023i002p00165.

Morrow, R., and P.-Y. Le Traon, 2012: Recent advances in observing mesoscale ocean dynamics with satellite altimetry. Adv. Space Res., 50, 1062-1076, doi:10.1016/j.asr.2011.09. 033.

Poje, A., and Coauthors, 2014: Submesoscale dispersion in the vicinity of the Deepwater Horizon spill. Proc. Natl. Acad. Sci. USA, 111, 12 693-12 698, doi:10.1073/pnas.1402452111.

Ponte, A., and P. Klein, 2013: Reconstruction of the upper ocean 3D dynamics from high-resolution sea surface height. Ocean Dyn., 63, 777-791, doi:10.1007/s10236-013-0611-7.

- —, X. Capet, P.-Y. Le Traon, B. Chapron, and P. Lherminier, 2013: Diagnosing surface mixed layer dynamics from high-resolution satellite observations: Numerical insights. J. Phys. Oceanogr., 43, 1345-1355, doi:10.1175/ JPO-D-12-0136.1.

Pujol, M.-I., G. Dibarboure, P.-Y. Le Traon, and P. Klein, 2012: Using high-resolution altimetry to observe mesoscale signals. J. Atmos. Oceanic Technol., 29, 1409-1416, doi:10.1175/ JTECH-D-12-00032.1.

Rhines, P. B., 1975: Waves and turbulence on a beta-plane. J. Fluid Mech., 69, 417-443, doi:10.1017/S0022112075001504.

Richman, J. G., B. K. Arbic, J. F. Shriver, E. J. Metzger, and A. J. Wallcraft, 2012: Inferring dynamics from the wavenumber spectra of an eddying global ocean model with embedded tides. J. Geophys. Res., 117, C12012, doi:10.1029/ 2012JC008364. 
Roullet, G., and P. Klein, 2010: Cyclone-anticyclone asymmetry in geophysical turbulence. Phys. Rev. Lett., 104, 218501, doi:10.1103/PhysRevLett.104.218501.

Shafer Smith, K. S., and G. K. Vallis, 2001: The scales and equilibration of midocean eddies: Freely evolving flow. J. Phys. Oceanogr., 31, 554-571, doi:10.1175/1520-0485(2001)031<0554: TSAEOM $>2.0 . \mathrm{CO} ; 2$.

Shchepetkin, A. F., and J. C. McWilliams, 2005: The regional oceanic modeling system (ROMS): A split-explicit, free-surface, topography-following-coordinate oceanic model. Ocean Modell., 9, 347-404, doi:10.1016/j.ocemod.2004.08.002.

Vallis, G. K., 2006: Atmospheric and Oceanic Fluid Dynamics: Fundamentals and Large-Scale Circulation. Cambridge University Press, 745 pp.

$\mathrm{Xu}$, Y., and L.-L. Fu, 2012: The effects of altimeter instrument noise on the estimation of the wavenumber spectrum of sea surface height. J. Phys. Oceanogr., 42, 2229-2233, doi:10.1175/ JPO-D-12-0106.1. 